Supporting Information for

Solving the Thermoelectric Trade-Off Problem with Metallic Carbon Nanotubes

Yota Ichinose, ${ }^{1}$ Akari Yoshida, ${ }^{1}$ Kanako Horiuchi, ${ }^{1}$ Kengo Fukuhara, ${ }^{1}$ Natsumi Komatsu, ${ }^{3}$ Weilu Gao, ${ }^{3}$ Yohei Yomogida, ${ }^{1}$ Manaho Matsubara, ${ }^{2}$ Takahiro Yamamoto, ${ }^{2}$ Junichiro Kono, ${ }^{* 3,4,5}$ and Kazuhiro Yanagi*1

${ }^{1}$ Department of Physics, Tokyo Metropolitan University, Tokyo 192-0372, Japan

${ }^{2}$ Department of Liberal Arts, Faculty of Engineering, Tokyo University of Science, Katsushika, Tokyo 125-8585, Japan

${ }^{3}$ Department of Electrical and Computer Engineering, Rice University, Texas 77005, USA

${ }^{4}$ Department of Physics and Astronomy, Rice University, Texas 77005, USA

${ }^{5}$ Department of Materials Science and NanoEngineering, Rice University, Texas 77005, USA

Corresponding Authors;

Junichiro Kono, E-mail: kono@rice.edu, Phone: +1-713-348-2209

Kazuhiro Yanagi, E-mail: yanagi-kazuhiro@tmu.ac.jp, Phone: +81-42-677-2494 


\section{Sample Characteristics}

a

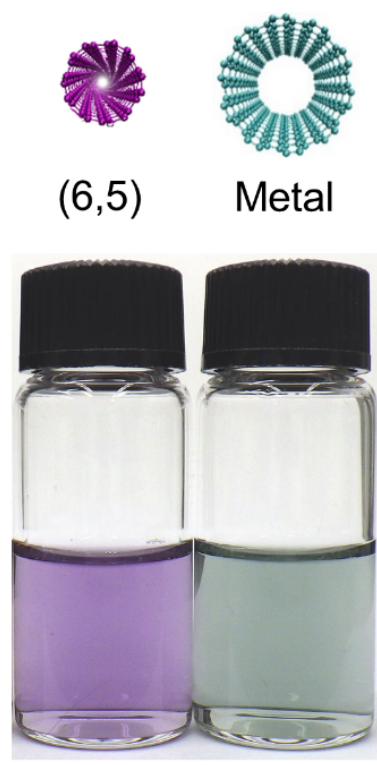

b

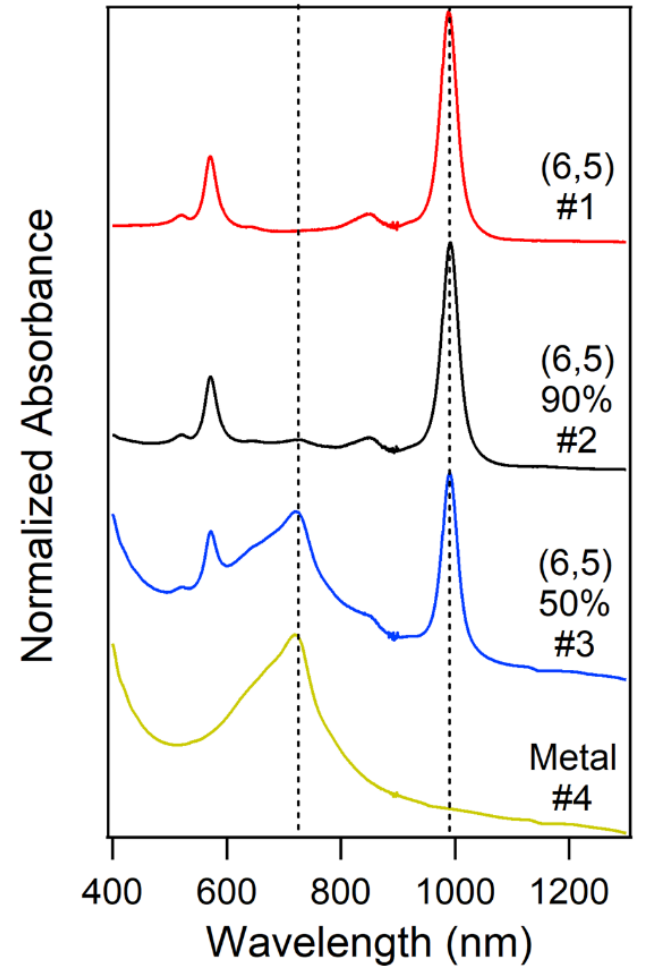

Figure S1. Sample information. a, A picture of aqueous solutions of purified SWCNTs used for making thin films. They are enriched in $(6,5)$ SWCNTs (left) and metallic SWCNTs (right), respectively. b, Optical absorbance spectra for Samples 1-4 (see Table 1 in the main text). We systematically changed the (6,5)-metal ratio - \#1: 100\%-0\%, \#2 90\%-10\%, \#3: 50\%-50\%, and \#4: 0\%-100\%. 

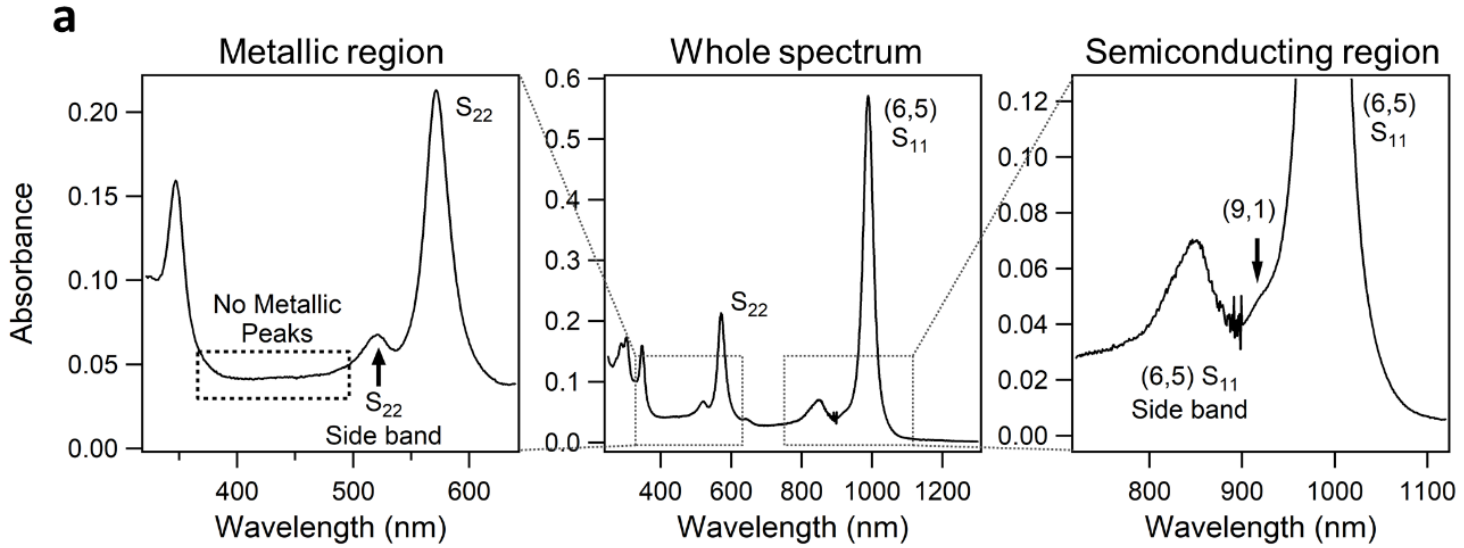

b

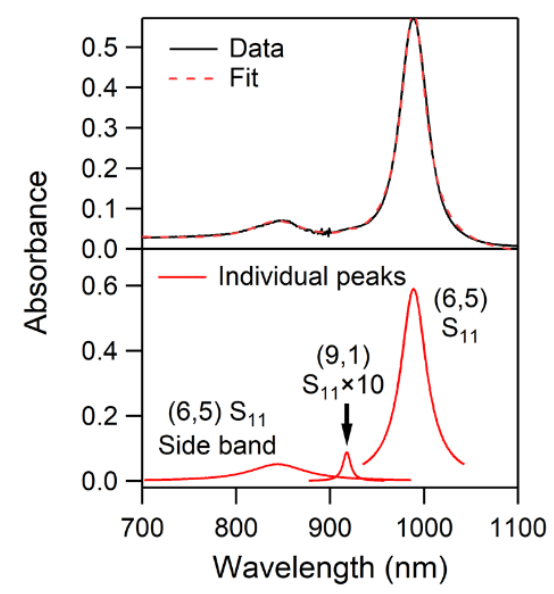

C

\begin{tabular}{|l|c|r|r|c|}
\hline Chirality & $(6,5)$ & \multicolumn{1}{|c|}{$(9,1)$} & \multicolumn{1}{c|}{ Metal } & \multicolumn{1}{c|}{ Total } \\
\hline Area & 30.838 & 0.139 & 0.0 & 30.997 \\
\hline Ratio & $99.5 \%$ & $0.5 \%$ & $0.0 \%$ & $100 \%$ \\
\hline
\end{tabular}

Figure S2. Purity evaluation for the high-purity $(6,5)$ sample (Sample 1 in Table 1 in the main text). a, Optical absorbance spectrum. The middle panel shows the whole spectrum in a range of $200-1300 \mathrm{~nm}$. The left panel shows a part of the spectrum in a range of $\sim 300-600$ $\mathrm{nm}$ (the "metallic" region). If metallic SWCNTs exist in the sample, they should exhibit peaks at $400 \mathrm{~nm}$ and $470 \mathrm{~nm}$. However, in this region, the spectrum is completely flat. Therefore, there are no detectable metallic SWCNTs in this sample. ${ }^{1}$ The right panel shows a part of the spectrum in a range of $\sim 700-1100 \mathrm{~nm}$ (the "semiconducting" region), which has several semiconducting peaks. A peak at $850 \mathrm{~nm}$ is the phonon side band of $(6,5) \mathrm{S}_{11}$. A shoulder at 910 $\mathrm{nm}$ is the absorbance of $(9,1) \mathrm{S}_{11}$. $\mathbf{b}$, Fitting results in the semiconducting region. The upper panel shows both the fit and absorbance spectra in the semiconducting region. The lower panel shows the individual peaks used for fitting. c, Comparison of peak areas. Each peak area value was obtained from the fit. As a result, the area of $(6,5)$ accounted for $99.5 \%$ of the total area. Since we cannot quantitatively evaluate the purity beyond $99 \%$ using absorbance spectra, the $(6,5)$ purity can be assumed to be more than $99 \%$. 


\section{Thermocouples}
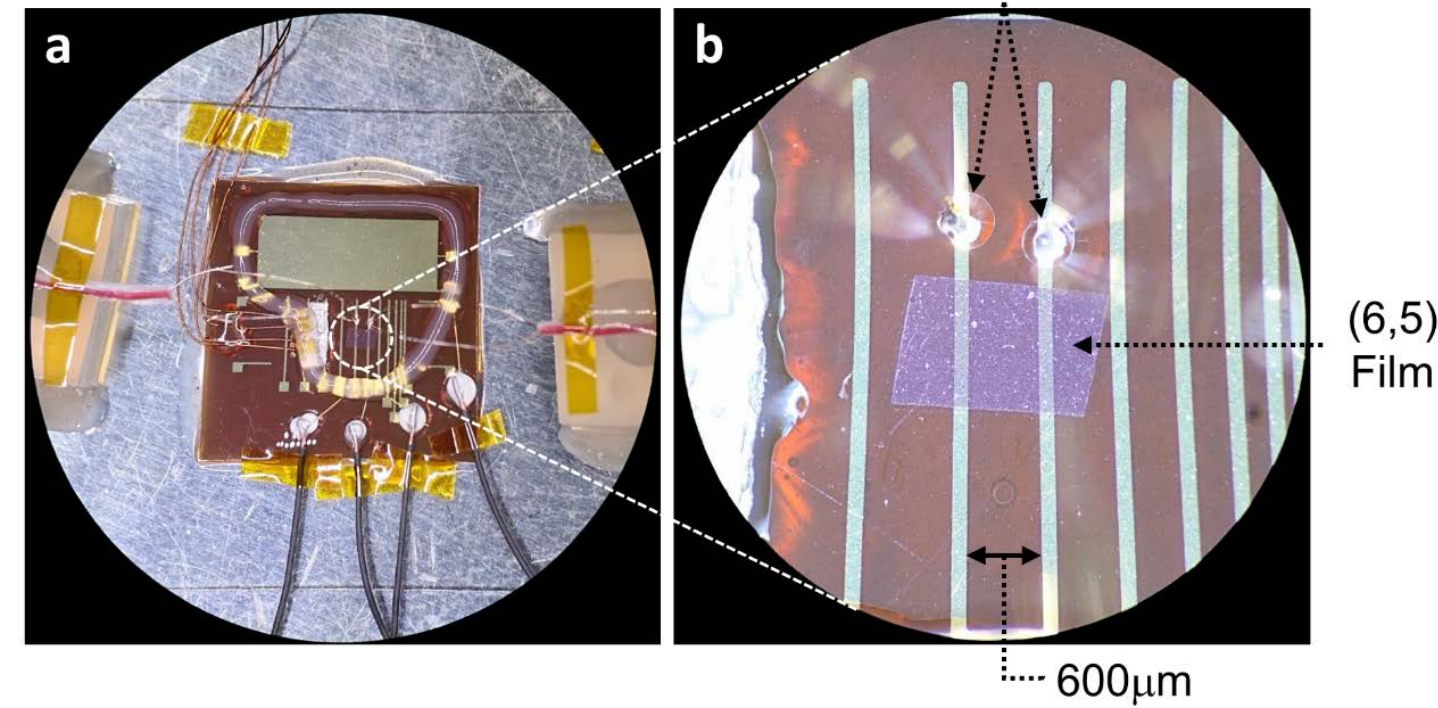

Figure S3. Device pictures. a, A picture of the whole device. $\mathbf{b}$, A picture zoomed in on a $(6,5)$ film. The temperature gradient and thermoelectric voltage was measured by thermocouples near both ends of the film. The distance between channels was $600 \mu \mathrm{m}$. 


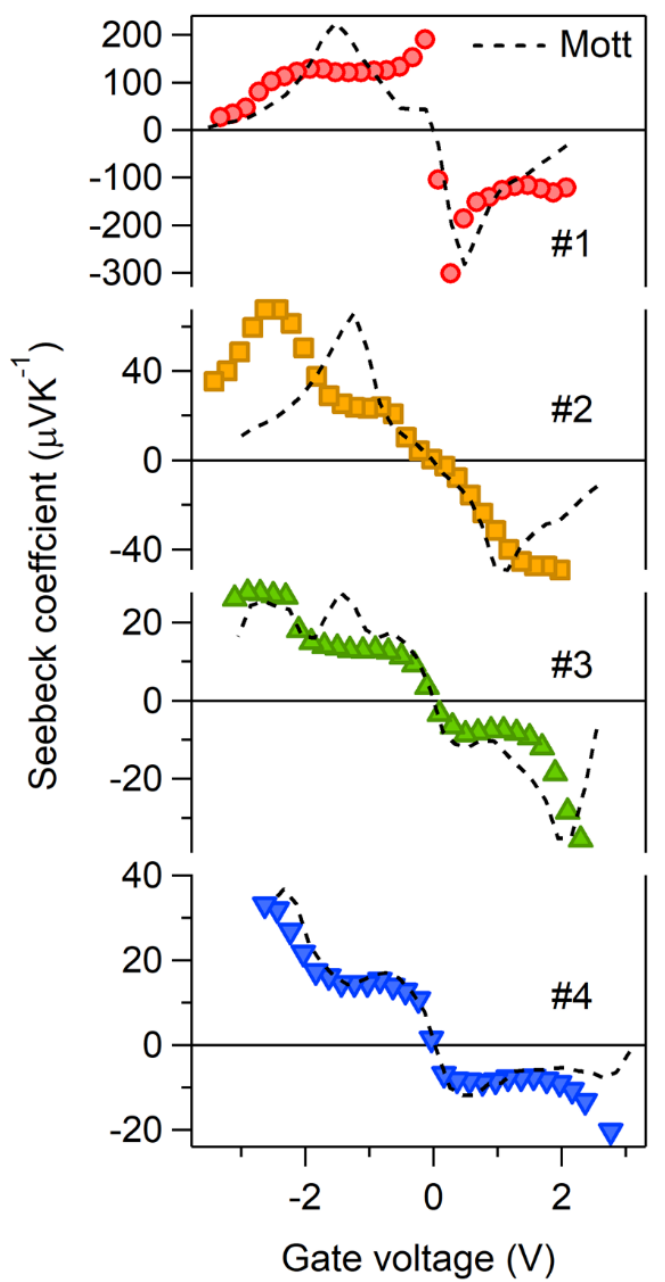

Figure S4. Comparison between experimental data and theoretical calculation by the Mott formula for Sample 1-4. See Table 1 in the main text for the semiconductor-metal ratios of the samples. Color points are the experimental data and black dashed lines are the results of calculations by the Mott formula (Equation (1) in the main text). 
a

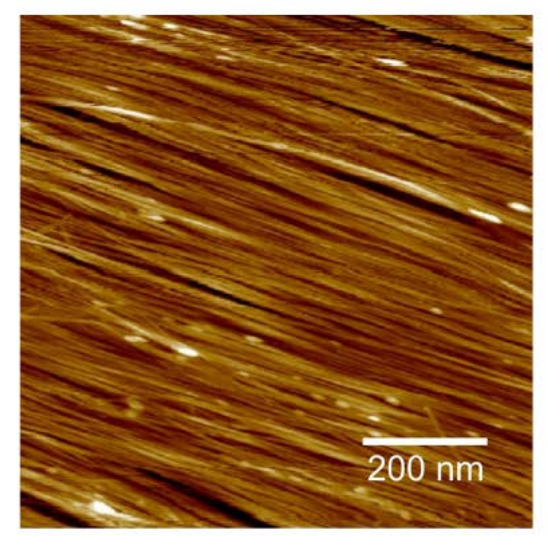

b

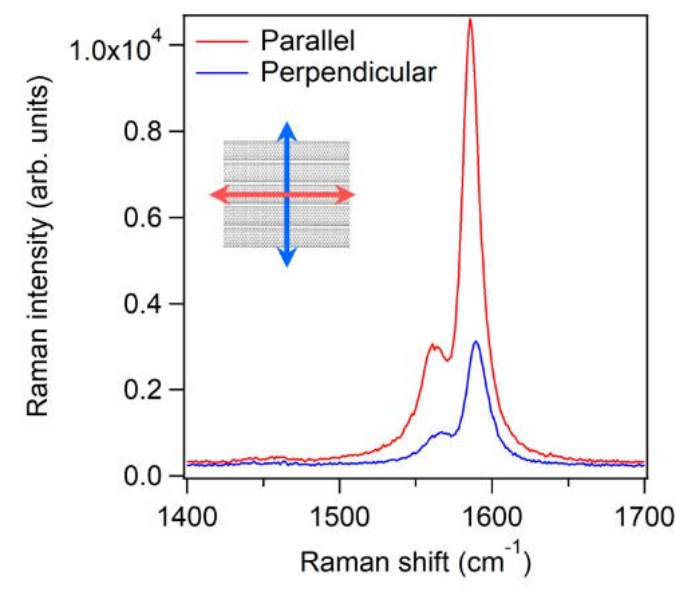

Figure S5. Information on the aligned metallic SWCNT film. a, A picture of an atomic force microscopy (MultiMode 8, Bruker Japan K.K.) image of an aligned metallic SWCNT film (Sample 5 in the main text). b, Raman spectra for the sample with excitation light polarized parallel (red line) and perpendicular (blue line) to the nanotube alignment axis. We identified the alignment direction and evaluated the alignment degree using polarized Raman measurements with a laser wavelength of $488 \mathrm{~nm}$. The average $I_{\|} / I_{\perp}$, which is the ratio of the $G$-band peak intensity for parallel polarization to that for perpendicular polarization, for the sample was $2.6 \pm 0.2$. 


\section{Theory of Thermoelectric Properties of SWCNTs}

\section{The details of theoretical calculations based on linear response theory combined with the thermal Green's function method within the constant-relaxation-time approximation.}

Here we explain the theoretical procedure we used to obtain the relationships between the Seebeck coefficient $S$ and the electrical conductivity $\sigma$ in semiconducting SWCNTs, which are indicated by dashed curves in Figure 3a. Within Kubo's linear response theory, $S$ as a function of $\sigma$ can be expressed as

$$
S=\frac{1}{T} \frac{L_{12}}{\sigma}
$$

Here, $\sigma$ and $L_{12}$ are given, respectively, by

$$
\sigma=\int_{-\infty}^{\infty} d E\left(-\frac{\partial f(E-\mu)}{\partial E}\right) \alpha(E)
$$

and

$$
L_{12}=-\frac{1}{e} \int_{-\infty}^{\infty} d E\left(-\frac{\partial f(E-\mu)}{\partial E}\right)(E-\mu) \alpha(E)
$$

where $e(>0)$ is the elementary charge, $\mu$ is the chemical potential, $f(E-\mu)$ is the Fermi-Dirac distribution function, and $\alpha(E)$ is the spectral conductivity.

The electronic states of the lowest conduction- and highest valence-band edges of a semiconducting SWCNT can be regarded as one-dimensional (1D) Dirac electrons. ${ }^{2}$ For 1D Dirac electrons, $\alpha(E)$ can be expressed as

$$
\alpha(E)=\frac{\hbar e^{2} v^{2}}{2 \pi V} \sum_{k} \operatorname{Tr}\left[\sigma_{x} G^{\mathrm{A}}(k, E) \sigma_{x} G^{\mathrm{R}}(k, E)-\operatorname{Re}\left\{\sigma_{x} G^{\mathrm{R}}(k, E) \sigma_{x} G^{\mathrm{R}}(k, E)\right\}\right]
$$

using the retarded/advanced Green's functions $G^{\mathrm{R} / \mathrm{A}}(k, E)$, where $v$ is the velocity of a Dirac electron, $V$ is the volume of the system, and $\sigma_{x}$ is the $x$ component of the Pauli matrices. Within the constant-relaxation-time approximation, the retarded/advanced Green's functions for a 1D Dirac electron are given by

$$
G^{\mathrm{R} / \mathrm{A}}(k, E)=\left\{\left(E \pm i \frac{\hbar}{2 \tau}\right) I-\left(\hbar v k \sigma_{x}+\Delta \sigma_{z}\right)\right\}^{-1}
$$

where $\tau$ is the relaxation time of a Dirac electron and $\sigma_{z}$ is the $z$ component of the Pauli matrices. Once $G^{\mathrm{R} / \mathrm{A}}(k, E)$ is calculated, we can calculate $\alpha(E)$ and eventually obtain both $S$ and $\sigma$.

As mentioned in the main text of this paper, the appearance of in-gap states due to the presence of metallic SWCNTs in a semiconducting SWCNT film can be represented by the relaxation time $\tau$. In fact, the in-gap DOS increases with decreasing $\tau$. Thus, the experimental data of $S$ and $\sigma$ for semiconducting SWCNTs including various amounts of metallic SWCNTs can be theoretically fit by choosing adequate $\tau$. 
The details of theoretical calculation on thermoelectric properties of single $(6,5)$ and $(10,10)$ SWCNTs.

For theoretical comparison (Figure 4 in the main text) between the power factor of single $(6,5)$ and $(10,10)$ SWCNTs, we performed the following calculation. Within the framework of the Landauer formula, the conductance $G(\mu)$ and Seebeck coefficient $S(\mu)$ with the chemical potential $\mu$ are expressed as

$$
G(\mu)=\frac{2 q^{2}}{h} \int d E T(E)\left(-\frac{\partial f(E, \mu)}{\partial E}\right)
$$

and

$$
S(\mu)=\frac{1}{q T} \frac{\int d E T(E)\left(-\frac{\partial f(E, \mu)}{\partial E}\right)(E-\mu)}{\int d E T(E)\left(-\frac{\partial f(E, \mu)}{\partial E}\right)},
$$

where $q$ is the charge of carriers, $h$ is the Planck constant, $f(E, \mu)$ is the Fermi-Dirac distribution function and $T(E)$ is the transmission function. As can be seen here, we can obtain $G(\mu)$ and $S(\mu)$, once $T(E)$ is calculated.

We used the tight-binding method to calculate the thermoelectric properties of isolated $(6,5)$ and $(10,10)$ SWCNTs with $1 \times 1 \times 9$ Monkhorst-Pack K-grid. The Slater-Koster parameters used in this method were obtained by the density-functional tight-binding method ${ }^{3}$ utilizing the "hotbit" code. ${ }^{4}$ Under these parameters we performed the calculation of density of states (DOS) for bulk SWCNTs. In addition, $T(E)$ of SWCNTs was calculated by combing the nonequilibrium Green's function method with the tight-binding method. SWCNTs were divided into three regions; device region, a left and a right electrode. The length of device region is 5 unit cell for $(6,5)$ SWCNT and 10 unit cell for $(10,10)$ SWCNT. The above-mentioned calculation was implemented in the ATK-SE package (Ver. 2019.3). ${ }^{5}$ The calculated conductance, Seebeck coefficient, power factor, and density of states (DOS) for $(6,5)$ and $(10,10)$ SWCNTs are depicted below (Figure S6). 
a
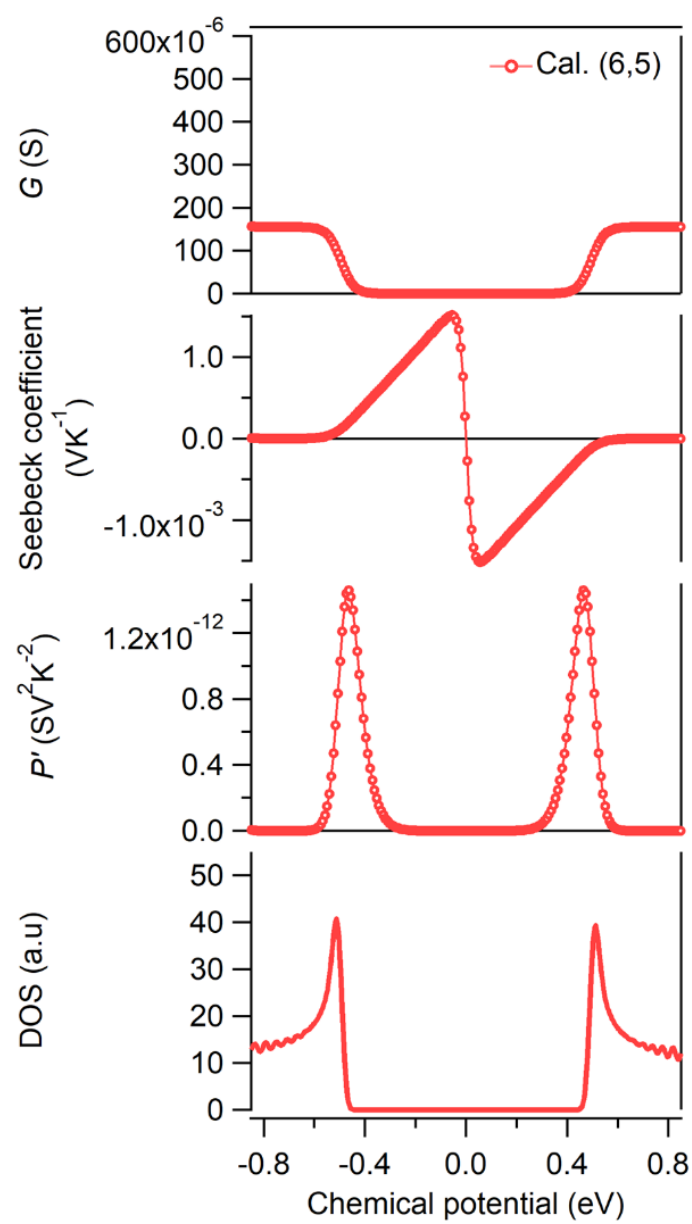

b

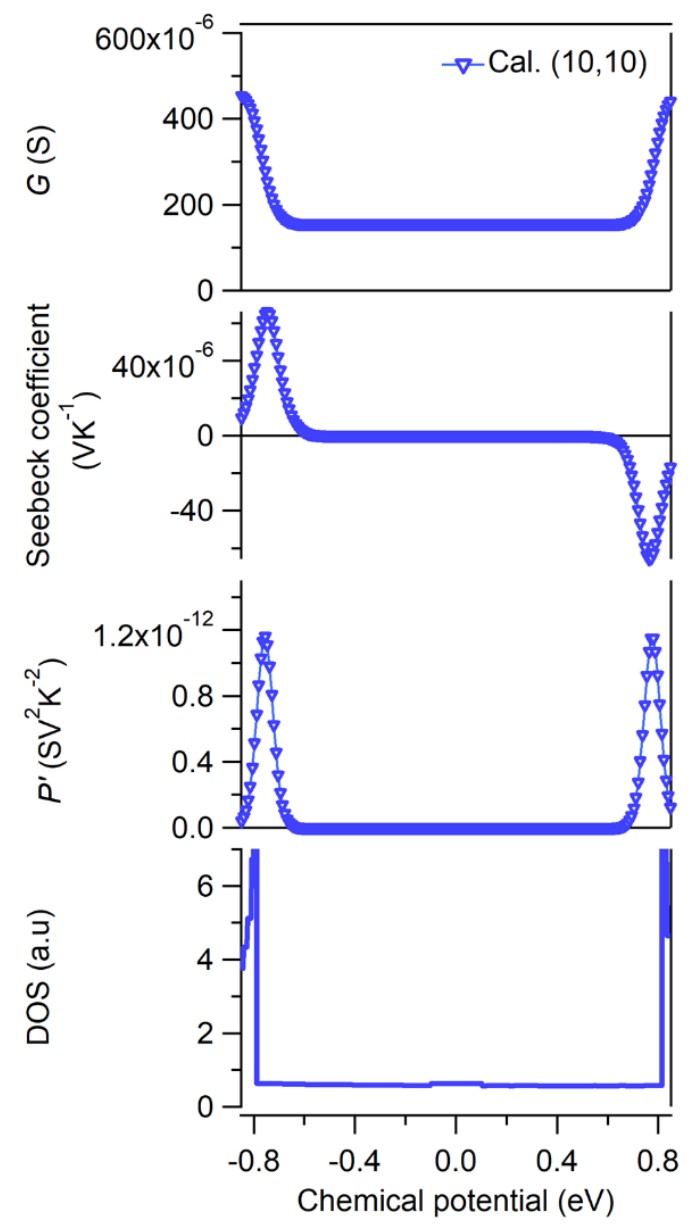

Figure S6. Calculated conductance, Seebeck coefficient, power factor, DOS of $(6,5)$ and $(\mathbf{1 0 , 1 0 )}$ SWCNTs. Calculated conductance, Seebeck coefficient, power factor and DOS of a, $(6,5)$ and $\mathbf{b},(10,10)$ SWCNT are shown as a function of chemical potential. Figure 4 in the main text is based on these plots. 


\section{References}

1. Ichinose, Y.; Eda, J.; Yomogida, Y.; Liu, Z.; Yanagi, K., Extraction of High-Purity SingleChirality Single-Walled Carbon Nanotubes through Precise Ph Control Using Carbon Dioxide Bubbling. J. Phys. Chem. C 2017, 121, 13391-13395.

2. Yamamoto, T.; Fukuyama, H., Bipolar Thermoelectric Effects in Semiconducting Carbon Nanotubes: Description in Terms of One-Dimensional Dirac Electrons. J. Phys. Soc. Jpn. 2018, 87, 114710.

3. Koskinen, P.; Mäkinen, V., Density-Functional Tight-Binding for Beginners. Comput. Mater. Sci. 2009, 47, 237-253.

4 https://github.com/pekkosk/hotbit

5. Stokbro, K.; Petersen, D. E.; Smidstrup, S.; Blom, A.; Ipsen, M.; Kaasbjerg, K., Semiempirical Model for Nanoscale Device Simulations. Phys. Rev. B 2010, 82, 075420. 